\title{
El gabinete de comunicación del hospital. Propuesta teórica y acercamiento a la realidad de los departamentos de comunicación de los hospitales públicos de Galicia
}

\section{The Hospital Communication office. Theoretical proposal and approach to the reality of the communication offices of public hospitals in Galicia}

\section{Carmen Costa Sánchez. Universidade da Coruña}

Recibido: 20-XII-2010 - Aceptado: 6-IX-2011

Resumen:

La comunicación en el sector hospitalario constituye una herramienta imprescindible para conseguir una imagen positiva interna-externa y en relación con los usuarios. El presente artículo tiene como objetivo principal analizar la situación de los departamentos de comunicación en los hospitales públicos de Galicia. Para ello se han combinado metodologías cuantitativas (cuestionarios) y cualitativas (entrevista personal con sus profesionales). Los resultados muestran una situación más favorable para los hospitales de mayor tamaño. Aun así, los recursos humanos son pocos, la comunicación externa es la apuesta principal y todavía queda mucho camino a la hora de concebir la comunicación como una herramienta estratégica de gestión.

Palabras clave:

Comunicación Institucional, Relaciones Públicas, Hospital, Galicia

Abstract:

The role of communication in the hospital sector is an essential tool to achieve a positive internal-external image and a good relationship with users. The objective of the present article is to analyse the situation of communication offices in the Galician public hospitals. We have combined quantitative (questionnaires) and qualitative (personal interview with his professionals) methodologies. The results show a more favourable situation for the hospitals of greater size. Nevertheless the human resources are few, the External Communication is the main activity and it still remains a long way to conceive the communication like a strategic tool of management.

Keywords:

Institutional Communication, Public Relations, Hospital, Galicia

doxa.comunicación | n 14 | 175 


\section{El lugar de la Comunicación en el servicio hospitalario. Identificación de las necesidades comunica- tivas del hospital}

Cada organización -cada empresa y cada institución- presentan una serie de particularidades vinculadas al sector en el cual trabajan, al tipo de actividad que desarrollan, al tipo de profesionales que necesitan, a la variedad y tipología de públicos (internos y externos) a los que se dirigen, que inciden en la política comunicativa que llevan a cabo.

La institución hospitalaria se dedica a la prestación de un servicio prioritario que consiste en la correcta atención sanitaria (preventiva, curativa y rehabilitadora) de un grupo de población que así lo requiere. Para la prestación de dicho servicio se necesitan cierto tipo de productos: una radiografía, unos análisis, una receta... que son instrumentos intermediarios necesarios para la prestación del servicio, pero que no constituyen el servicio en sí mismo.

El servicio que presta el hospital es un servicio diferente al que proporcionan otras empresas o instituciones, primeramente, porque la necesidad que lo origina, la de rehabilitación y vigilancia del estado de salud de la población, es una necesidad que podemos caracterizar como:

- Básica, que no se puede eludir o aplazar si la persona está enferma y esto le impide continuar su vida con normalidad.

Garantizada por el Estado, al menos en España, por lo que se paga indirectamente a través de los impuestos (existe, no obstante, la posibilidad de acudir a un centro hospitalario privado).

- Universal, pues personas de todas las edades y de ambos géneros precisarán dar respuesta a esta necesidad en algún momento de su vida.

En la clasificación de los servicios propuesta por Ruiz (1990), el servicio hospitalario sería un servicio puro de alto grado de contacto. Los servicios puros son aquellos en los que el servicio es la entidad primaria que se vende u ofrece. Los servicios de alto grado de contacto implican la constante presencia del cliente en el proceso de prestación del servicio. Los servicios de alto grado de contacto presentan cuatro características generales (Ruiz, 1990):

Están más sujetos a incertidumbre en las operaciones de rutina, desde el momento en que el usuario puede intervenir o algo puede producir una irrupción en cualquier momento.

- $\quad$ Es muy raro que en los servicios de alto contacto se disponga de una capacidad adecuada para dar respuesta a la demanda cuando se presentan ciertos picos. En general, se podrá calcular su aparición por experiencia o análisis estadístico dentro de un grado de probabilidad que no es exacto (ej. Pensemos en el "calendario" de los hospitales y centros de atención primaria que provoca que la gripe sature las urgencias en una determinada época del año). 
Se requiere de una dotación de personal con características destacadas en el área de relaciones humanas o públicas, característica de especial relevancia en materia de comunicación.

Los servicios de alto grado de contacto están más a merced del factor tiempo que los de bajo grado de contacto.

En el caso del servicio prestado en contexto de hospitalización, el servicio sanitario se completa con servicios adicionales para los pacientes que requieren ser ingresados (ubicación, instalaciones, comidas, comodidad, etc.). Además está lo que bautizamos como "servicio catalizador", es decir, la comunicación entre el personal y el usuario, que constituye un plus que se le proporciona al cliente y que revaloriza el servicio. Tal y como explica Costa (1995: 163), los cuidados hospitalarios son tan importantes como las curas hospitalarias, e incluso la atención y la información que reciben los familiares del paciente son tan importantes como el servicio, ya que llegan a formar parte de él. Esto supone que los individuos quieren que se los trate como tales, y no como objetos ni como sujetos de la burocracia administrativa.

En el servicio hospitalario global confluyen: el servicio médico (la restauración/ comprobación del estado de salud del usuario); los servicios complementarios (de alojamiento, de limpieza, de comidas...), que ganan importancia en caso de ingreso en el hospital; y el servicio catalizador (la comunicación). La comunicación constituye el elemento que aporta valor añadido al servicio hospitalario, pues a través de la comunicación puede adaptarse el servicio a la idiosincrasia y necesidades de cada paciente.

La comunicación, por lo tanto, forma parte del servicio que se le presta al usuario del hospital en diversos sentidos:

- $\quad$ Como base de la relación entre los profesionales sanitarios y el usuario y, por lo tanto, como parte del Servicio Médico: entrevista para la redacción de la historia clínica, prescripción de medicamentos, explicación de cuidados al alta, etc.

- Como parte del servicio “identificable” por el usuario: este puede desconocer los pormenores médicos del servicio, pero sabe si considera que fue bien o mal tratado y bien o mal informado.

- Como elemento que facilita su adaptación al proceso de hospitalización, contribuyendo a incrementar su satisfacción con el servicio global prestado.

- Como elemento educativo, pues constituye una importante herramienta para ayudar a convertir el hospital en una institución eficiente en la promoción de hábitos saludables (como parte de la estrategia de comunicación para la salud del hospital).

Todo esto redundará en una mejora de la calidad percibida ${ }^{1}$ por los usuarios. Una comunicación bien gestionada permitirá al hospital poner en marcha una triple estrategia de diferenciación, personalización y humanización. "El usuario de

1 La “calidad percibida”, según FONT es la "satisfacción del usuario respecto al servicio recibido. Componente humanitario que integra

doxa.comunicación | $n^{\circ} 14$ 
cualquier servicio sólo queda satisfecho si obtiene lo que desea cuando lo desea y en la forma que lo desea" (Corella, 1998: 155), una máxima que garantiza el éxito de organizaciones de servicios como el hospital.

\section{Propuesta de departamento de comunicación para un hospital.}

Las necesidades comunicativas internas y externas del hospital lo conducen, necesariamente, a la creación de un departamento encargado de diseñar, planificar, ejecutar, evaluar y coordinar la estrategia comunicativa -hacia adentro y hacia fuera- que desea llevarse a cabo. Con este objetivo fueron naciendo en los hospitales gabinetes de comunicación, aunque en España la creación de estos departamentos se inició hace apenas dos décadas. Según González Borjas (2004: 308), los hospitales públicos comenzaron a incorporar en la década de los 90 la gestión de la comunicación entre sus prioridades al objeto de llegar de forma más directa y nítida a los ciudadanos y, paralelamente, obtener los beneficios de una imagen positiva de la gestión realizada.

La conciencia de la necesidad de un departamento de comunicación y su papel central en el ámbito hospitalario depende de la filosofía de cada hospital y de su equipo de gobierno, aunque la tendencia confirma que son cada vez menos los hospitales que carecen de un departamento que organice y planifique su estrategia comunicativa:

"Hoy el gerente del hospital ya tiene asumida la necesidad de comunicación como un instrumento valioso de la gestión, el porqué y para qué la precisa: sabe que el desarrollo de una organización de estilo participativo debe contar con un sistema de comunicación interna. También es consciente de que para hacer efectiva una política que haga del usuario/cliente el verdadero eje de la organización, las técnicas comunicacionales juegan un papel indispensable. Y finalmente está convencido de que la organización tiene que extravertirse, que un hospital no es una isla ni un fortín; debe integrarse en la comunidad a la que sirve y de la que forma parte, sintonizando con sus intereses y demandas, y para ello debe vertebrarse un sistema de comunicación con sus públicos externos" (ACSAS, 1999: 99).

Con el término "Comunicación Hospitalaria” denominamos a aquella cuya titularidad corresponde al Hospital (sea público o privado, tenga finalidad lucrativa o no), quien la diseña, planifica, ejecuta y evalúa con finalidad estratégica a través de su Gabinete o Departamento de Comunicación, en sus vertientes interna, intermedia y externa, con la finalidad de darse a conocer socialmente en términos positivos y de servir y contribuir desde la faceta comunicadora al bienestar de sus profesionales, de sus usuarios y de la sociedad en general. Las vertientes de la Comunicación Hospitalaria serán, por lo tanto tres: hacia los públicos externos, hacia los públicos internos, y hacia los públicos intermedios (entendiendo como tales los usuarios efectivos de los servicios del hospital, lo que incluye a pacientes y familiares de pacientes o acompañantes).

el trato, la información, las condiciones ambientales, las instalaciones y la hostelería. Suele ser la dimensión más valorada por el paciente-usuario", en Asenjo Sebastián, M. A. (2006) (dir.): Gestión diaria del hospital, Barcelona: Masson, p. 383. 
Sin embargo, los profesionales de los departamentos de comunicación hospitalarios se encuentran, a menudo, en un sector que relega sus funciones a las relaciones con los medios, infravalorando su potencial al servicio del plan estratégico del hospital ${ }^{2}$.

Desde nuestro punto de vista, el gabinete de comunicación del hospital es el departamento que debe ocuparse de la planificación de la comunicación de la institución. Para eso, jerárquicamente, debería depender del órgano máximo de gestión, es decir, la Gerencia. De esta forma, se garantiza que el gabinete tenga contacto con los máximos representantes de la institución; tenga conocimiento del contexto para una orientación correcta de la comunicación; tenga conocimiento de las estrategias reales de la organización (acciones públicas o no) para un correcto diseño y orientación de las estrategias de comunicación y de los mensajes a transmitir.

El departamento de comunicación hospitalario tendrá una función esencial en las áreas de comunicación interna y de comunicación al usuario. En el primer caso, debido a la variedad de perfiles profesionales que aglutina el hospital y al papel de embajadores de la institución que estos desempeñan ante el usuario. En el segundo, como supervisor de las tareas de comunicación dirigidas a los usuarios efectivos de los servicios del hospital:

"A este profissional de Comunicação compete a gestão e administração dos aspectos relativos á imagem institucional, relações com a comunidade circundante, ligação á comunicação social, informação e orientação dos utentes (doentes e familiares) do hospital, recepção e tratamento de reclamação, assim como os aspectos relativos ao marketing institucional, de acordo com as políticas definidas pelo CA em profunda articulação com as restantes direccções. Compete-lhe ainda a responsabilidade sobre as unidades funcionais, habitualmente, dele directamente dependentes -gabinete do utente / gabinete de informação e orientação central telefónica, recepção e serviço informativo” (Kuteev-Moreira, 2003: 6).

Basándonos en distintos autores ${ }^{3}$, proponemos como funciones del modelo de Departamento de Comunicación de un Hospital, las siguientes:

2 “Todavía no se reconoce al periodista dentro del hospital”, entrevista a María Pilar Muñoz, responsable del Gabinete de Comunicación del Hospital Universitari La Fe de Valencia en PrNoticias: http://www.prnoticias.es/content/view/10019718/227/ [Consultado el $02 / 12 / 08]$.

3 Martínez Ortuño, I. (1994): “Modelo comunicativo para un hospital”, en Orive Riva, P., Comunicación sanitaria, Madrid: Dossat 2000, pp. 78-83; March Cerdá, J .C. et al. (2002): “Marketing y comunicación interna en hospitales y en atención primaria: opinión de profesionales y de gestores”, en Horizonte sanitario, no 1, pp. 6-17; Kuteev-Moreira, P. (2003): “O Gabinete de Comunicação no Hospital”, en Comunicação Empresarial, nº 25, pp. 4-9. 
Figura 2. Modelo de departamento de comunicación para un hospital

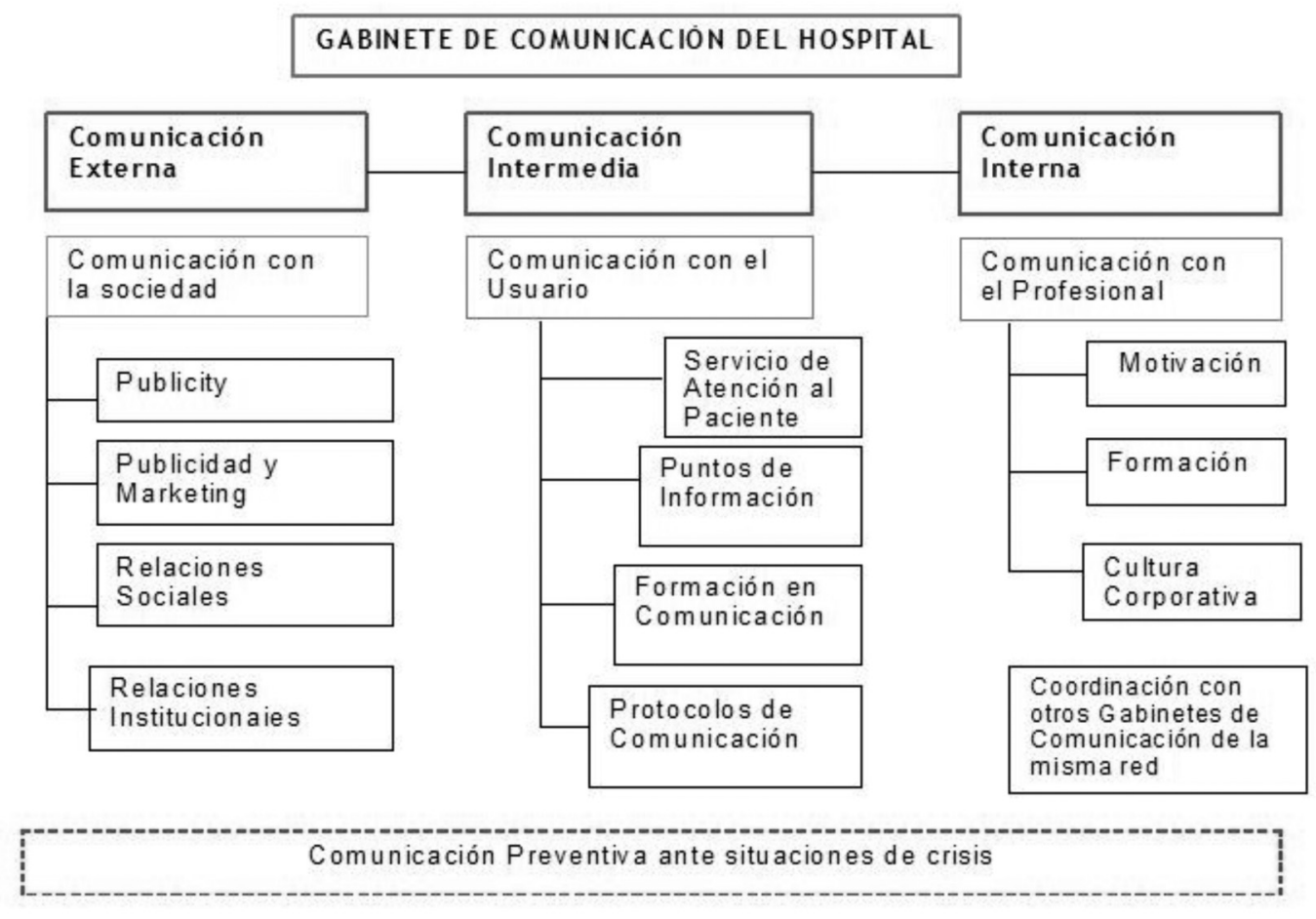

Elaboración propia

\section{La Comunicación Hospitalaria Pública en Galicia: Estado de la cuestión}

\subsection{Metodología aplicada}

La metodología aplicada engloba el uso de técnicas cuantitativas y cualitativas. Las técnicas cuantitativas se basaron en el empleo de dos cuestionarios: uno superficial para conocer qué hospitales públicos tenían departamento de comunicación y uno en profundidad para conocer las funciones de aquellos que existían. El cuestionario superficial permitió establecer un mapa general de hospitales con gabinete de comunicación en Galicia. 


\section{Cuestionario superficial empleado}

1.¿Existe en su hospital un gabinete o departamento de comunicación? Si / No

\section{2. ¿Qué nombre recibe dicho departamento?}

a) Gabinete de comunicación $\quad$ b) Dirección de comunicación $\quad$ c) Gabinete da prensa

d) Relaciones con los medios

e) Otro (especificar)

3. En caso de que lo haya, ¿cuántas personas lo componen? Total:_ No Hombres: _ No Mujeres: Distingan por favor entre hombres y mujeres

4. En caso de que no exista como tal, ¿̨hay alguien que desempeñe sus funciones?

a) No

b) Sí, se ocupa la/s misma/s persona/s de modo ocasional

c) Sí, se ocupa la/s misma/s persona/s con cierta regularidad

d) Sí, personas distintas en contadas ocasiones

e) Sí, personas distintas, pero con cierta regularidad

5. ¿A qué departamento pertenece/n esa/s persona/s?
a) Gerencia
b) Atención al usuario / cliente
c) Dirección Médica
d) Otros: (especificar)

6. ¿Qué actividad/es desempeña dicho gabinete o personal que ocupa sus funciones? (Las opciones no son excluyentes)
a) Mandar notas de prensa
b) Realizar un boletín de información o revista interna
c) Atender las solicitudes de información de los medios
d) Realizar el dossier de prensa de las noticias que hablen del hospital
e) Señaléctica del centro
f) Organización de eventos
g) Intranet
h) Otras: 
El cuestionario en profundidad permitió conocer de cerca todos los aspectos relacionados con el departamento de comunicación, sus funciones, relaciones jerárquicas, personal y actividades. Su elaboración parte del Modelo de departamento de comunicación para un hospital propuesto en el apartado anterior. En concreto se componía de los siguientes apartados:

A) Datos identificativos del Departamento de Comunicación:
Nombre del Hospital: Año de creación del gabinete:
Nombre del Gabinete:
No personas que integran el gabinete:
El Gabinete depende jerárquicamente de...
$\square$ Gabinete Consejería/SERGAS $\quad \square$ Gerencia del hospital $\quad \square$ Recursos Humanos $\square$ Otro

B) La formación del personal del Gabinete:

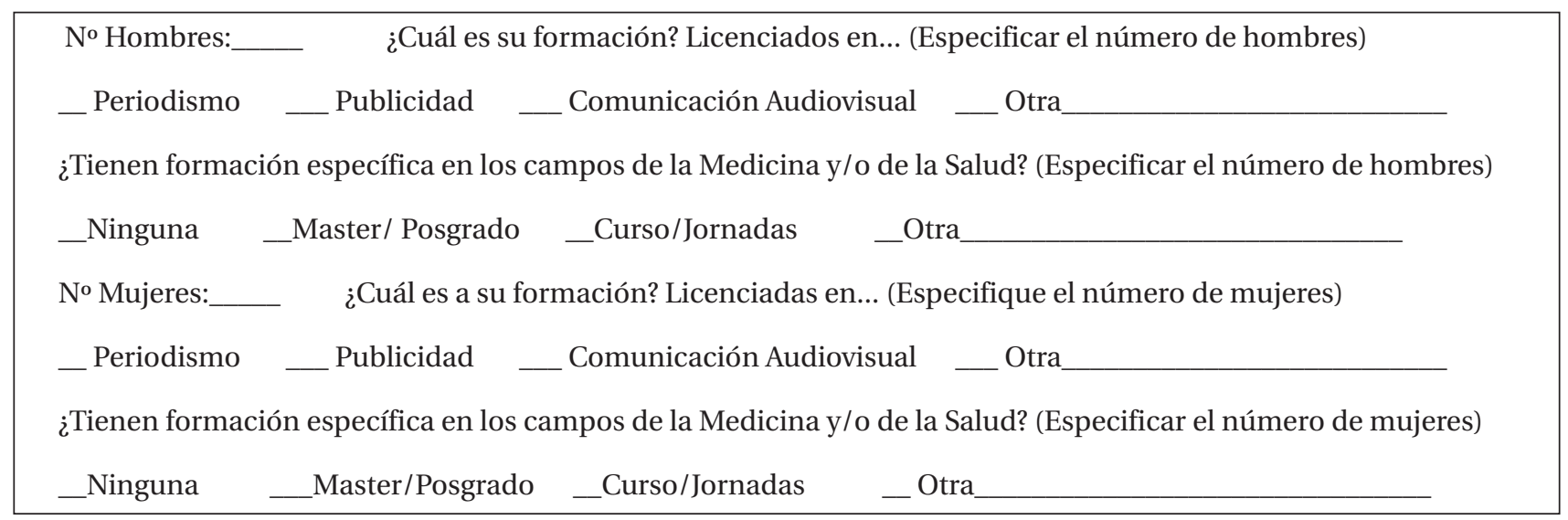

C) Las funciones del Gabinete respecto de la Comunicación Externa, Interna y con el Usuario:
¿Cuántas horas trabaja el Gabinete de media por día? .... h
¿Cuántas horas se dedican por día a...
Comunicación Externa
Comunicación Interna
Comunicación al Usuario

De 0 a 10, siendo 0 el mínimo y 10 el máximo, indique la importancia del trabajo del Gabinete con respecto a...

Comunicación Externa $\square 0 \quad \square 1 \quad \square 2 \square 3 \quad \square 4 \square 5 \square 6 \square 7 \square 8 \square 9 \square 10$ 


\section{Comunicación Interna $\square 0 \quad \square 1 \quad \square 2 \square 3 \quad \square 4 \quad \square 5 \square 6 \quad \square 7 \quad \square 8 \square 9 \square 10$ \\ Comunicación al Usuario $\square 0 \quad \square 1 \quad \square 2 \square 3 \quad \square 4 \square 5 \square 6 \square 7 \square 8 \quad \square 9 \square 10$}

Indique las funciones del Gabinete en Comunicación Externa...

$\square$ Relaciones con los medios de comunicación (notas y conferencias de prensa, demandas de información, etc.)

$\square$ Relaciones con otras instituciones (ej. Ayuntamiento, Universidad...)

$\square$ Relaciones con asociaciones de pacientes

$\square$ Actualización de las informaciones para la página web del hospital

$\square$ Manual de imagen corporativa

$\square$ Organización de actos/ eventos

$\square$ Mecenazgo

$\square$ Inserción de publicidad en los medios

$\square$ Otras:

Indique las funciones del Gabinete en Comunicación Interna...

$\square$ Planificación de reuniones informativas

$\square$ Planificación de encuentros informales entre el personal

$\square$ Actualización de contenidos en la Intranet

$\square$ Formación en comunicación del personal

$\square$ Manual de acogida a nuevos profesionales

$\square$ Tablón de anuncios

$\square$ Buzón de sugerencias para los públicos internos

$\square$ Documentos internos: memoria de actividades, circulares...

$\square$ Revista/Boletín interno

$\square$ Otras: 
Indique las funciones del Gabinete en Comunicación al Usuario...

$\square$ Redacción de materiales de información general: guía de acogida, campañas preventivas, etc.

$\square$ Redacción de materiales de información específica: formularios de Consentimiento Informado, instrucciones médicas, etc.

$\square$ Supervisión de los puntos informativos del Hospital

$\square$ Supervisión del Servicio de Atención al Paciente

$\square$ Supervisión de la señaléctica del centro

$\square$ Revista/Boletín para pacientes

$\square$ Otras:

D) Las relaciones entre el Departamento de Comunicación, el Servicio de Atención al Paciente y los Puntos Informativos del hospital

¿Qué relación mantienen el Gabinete de Comunicación y el Servicio de Atención al Paciente?

$\square$ Ninguna. Funcionan como departamentos independientes.

$\square$ El Servicio de Atención al Paciente depende del Gabinete de Comunicación.

$\square$ El Servicio de Atención al Paciente y el Gabinete de Comunicación comparten información

$\square$ Otra

No oficinas del Servicio de Atención al Paciente:__ N No Personal del Servicio de Atención al Paciente:

Dependencia jerárquica del Servicio: $\square$ Gerencia $\square$ Calidad $\square$ Recursos Humanos $\square$ Otra

No de puntos informativos del Hospital:

Dependencia jerárquica: : $\square$ Gerencia $\square$ SAP $\square$ Comunicación $\square$ Otra

Las entrevistas mantenidas con los profesionales de estos departamentos de comunicación (parte cualitativa) ayudaron a matizar y ampliar la información recogida gracias a los cuestionarios.

El universo de estudio lo formaron los 15 hospitales públicos de Galicia. Siete de ellos son complejos hospitalarios. Al ser tan reducido, universo y muestra son coincidentes. 


\subsection{Lectura inicial de los datos}

De los 15 hospitales y complejos hospitalarios públicos que existen en Galicia, un total de 6, todos ellos complejos hospitalarios, disponen de un departamento de comunicación interno, es decir, disponen de un departamento de comunicación ubicado en sus instalaciones: El Complejo Hospitalario Universitario de A Coruña ${ }^{4}$ (CHUAC), el de Santiago de Compostela (CHUS), el Complejo Hospitalario Arquitecto Marcide - Nóvoa Santos, el Complejo Hospitalario de Pontevedra (CHOP), el Complejo Hospitalario de Vigo (CHUVI) y el Complejo Hospitalario de Ourense (CHOU). Todos ellos reúnen las siguientes características:

Son complejos hospitalarios y como tales agrupan varios hospitales distintos y con distintas especialidades.

Todos son de nivel 3 en adelante, pues tienen más de 250 camas. Concretamente, tres son de nivel 1, pues tienen más de 1000 camas; dos son de nivel 2, entre 500 y 1000 camas, y uno es de nivel 3, con menos de 500.

- $\quad$ Todos tienen un amplio número de empleados que va desde los 1500 del Arquitecto-Marcide, el más pequeño, a los más de 5000 del Complejo Hospitalario Universitario de Vigo ${ }^{5}$.

El único complejo hospitalario que carece de gabinete de comunicación como departamento ubicado en dependencias hospitalarias es el Complejo Xeral-Calde de Lugo, que tiene una persona en el cuadro de personal del hospital, la cual fue trasladada a la Dirección Provincial del SERGAS en Lugo ${ }^{6}$, de manera que atiende la comunicación de los tres hospitales públicos de la provincia lucense (Complejo Hospitalario Xeral-Calde, Hospital da Costa y Hospital Comarcal de Monforte), además de la dirección provincial del SERGAS y de la Gerencia de Atención Primaria en esa provincia.

Cabe matizar a lo anteriormente expuesto, que el gabinete de comunicación del Complejo Hospitalario de Pontevedra está externalizado, pero dicho servicio se presta desde un departamento del hospital, con lo que, con independencia de que se trata de un tipo de contratación externo, la actividad resulta similar a la de los otros gabinetes de comunicación integrados por personal del SERGAS.

Por antigüedad, el departamento de comunicación del Complejo Hospitalario Universitario de A Coruña es el más antiguo, mientras que el departamento del Complejo Hospitalario Arquitecto Marcide - Nóvoa Santos es de reciente creación (septiembre de 2008).

Hasta el 2008, denominado Juan Canalejo.

Datos disponibles en la página web del SERGAS (Servizo Galego de Saúde): http://www.sergas.es

Los datos que se exponen en este apartado y las conclusiones que derivan de ellos fueron actualizados a septiembre de 2009 . 
Tabla I. Cronología de los gabinetes de comunicación de los hospitales públicos gallegos

\begin{tabular}{|c|c|c|c|c|c|c|}
\hline 1988 & 1992 & $1993^{7}$ & $1994^{8}$ & $1999^{9}$ & 2007 & 2008 \\
\hline & & & & & & Gabinete de \\
\hline Gabinete de & Gabinete de & Gabinete de & Gabinete de co- & Gabinete de & Gabinete de & Comunicación \\
\hline Comunicación & Comunicación & Comunicación & municación de & Comunicación & Comunicación & del Complexo \\
\hline del Complexo & del Complexo & de la Dirección & la Dirección & del Complexo & del Complexo & Hospitalario \\
\hline Hospitalario & Hospitalario & Provincial del & Provincial del & Hospitalario & Hospitalario & Arquitecto Mar- \\
\hline Universitario A & Universitario & SERGAS en Ou- & SERGAS en & Universitario & Pontevedra (Ex- & cide-Nóvoa \\
\hline Coruña & Vigo & rense & Lugo & Santiago & ternalizado) & Santos \\
\hline (CHUAC) & (CHUVI) & (CHOU) & (Xeral-Calde*) & (CHUS) & (CHOP) & $\begin{array}{l}\text { (Arquitecto } \\
\text { Marcide) }\end{array}$ \\
\hline
\end{tabular}

Fuente: Elaboración propia.

En cuanto a los recursos humanos de los gabinetes, oscilan entre una y dos personas (la gran mayoría son mujeres) y, con respecto a su formación, licenciadas en Ciencias de la Información o Periodismo.

En lo referente a sus funciones, vamos a realizar su análisis agrupando los departamentos por semejanzas en tres grandes grupos:

\subsubsection{CHUVI y CHOU: Gabinete externo e interno}

Entre el departamento de comunicación del Complejo Hospitalario Universitario de Vigo (CHUVI) y el de Ourense (CHOU) existen destacables paralelismos, que nos permiten agruparlos en una categoría para analizarlos conjuntamente:

7 En 1996, se incorpora la persona del equipo de comunicación que trabaja desde dentro del hospital.

8 Se incluye el gabinete de la Dirección Provincial del SERGAS en Lugo porque la creación de la plaza corresponde en realidad al cuadro de personal del Hospital Xeral-Calde y, posteriormente, no se creó ningún departamento interno. En el caso de Ourense, sucede algo parecido. Aunque tres años después, en 1996, comienza a trabajar una persona de Comunicación en las dependencias del hospital, se escoge la fecha de creación del gabinete de la Dirección Provincial porque desde ese año el gabinete empieza a ocuparse de la comunicación del hospital.

9 Según encontramos en Pereira, desde el año 1989, algunas personas pertenecientes por lo general al departamento de Gerencia se ocuparon de la Comunicación Externa del Hospital Xeral (no existía el complejo hospitalario como hoy lo conocemos), aunque en su contrato no figuraban esas funciones. En Pereira, R.: "La comunicación institucional de la salud en el ámbito local. El caso del complejo hospitalario universitario de Santiago de Compostela", en López Lita, R.; Fernández Beltrán, F. y Durán Mañes, A. (2006): La comunicación corporativa en el ámbito local, Castellón, Publicacions de la Universitat Jaume I. 
Así, en ambos complejos hospitalarios, una de las personas del gabinete trabaja dentro del hospital, pero la otra está ubicada físicamente en la Dirección Provincial del Sergas en la provincia correspondiente.

Su ubicación no es azarosa ni aleatoria. Implica que las personas ubicadas en las direcciones provinciales del SERGAS en esa provincia se ocupan de la comunicación de diversas entidades y constituyen, a nivel fáctico, los jefes de gabinete. En el caso de la periodista ubicada en la Dirección Provincial de Ourense, su actividad conlleva ocuparse de la comunicación externa de otras entidades hospitalarias, además del propio CHOU, en concreto:

- $\quad$ El Hospital de Verín (sito en la localidad de Verín, provincia de Ourense).

- $\quad$ El Hospital Comarcal de Valdeorras (sito en la localidad de O Barco de Valdeorras, provincia de Ourense).

Gerencia de Atención Primaria de Ourense.

La Dirección Provincial del Sergas en la provincia de Ourense.

La Delegación Provincial de la Consejería de Sanidad en Ourense.

En el caso de la periodista ubicada en la Dirección Provincial del SERGAS en Pontevedra, su actividad conlleva ocuparse de la comunicación externa del CHUVI y de las siguientes entidades:

La Dirección Provincial del Sergas en la provincia de Pontevedra.

Gerencia de Atención Primaria en Vigo.

La Delegación Provincial de la Consejería de Sanidad en Pontevedra en determinadas ocasiones ${ }^{10}$.

Las personas ubicadas en dependencias hospitalarias están al servicio, exclusivamente, de las necesidades comunicativas de ese hospital. Dada la división de funciones entre los ámbitos de comunicación externa e interna, las demandas de información de los periodistas se dirigen normalmente a quien está en la dirección provincial (quien les envía las notas de prensa), pero la persona de dentro es quien localiza las fuentes (médicos y otras) que van a dialogar con los periodistas. Esto implica una necesaria coordinación entre ambas personas en el ejercicio de su trabajo diario.

En cuanto a sus funciones en las distintas vertientes comunicativas, encontramos:

A) Enmarcadas como parte de la Comunicación Externa, las funciones de ambos gabinetes son semejantes: Relaciones con los medios de comunicación, Relaciones con otras instituciones, Relaciones con asociaciones de pacientes, Actualización de las informaciones de la página web del hospital, Manual de imagen corporativa y Organización de eventos.

10 Existe un periodista ocupado de la comunicación de la Delegación Provincial de la Consejería de Sanidad en Pontevedra, no obstante, en determinadas ocasiones puede suceder que se ocupe de la Comunicación Externa de alguna actuación que se produzca en el sur de la provincia (área de Vigo).

doxa.comunicación | n 14 
B) Como parte de las funciones de Comunicación Interna, existen diferencias respecto de la formación en comunicación del personal y de la planificación de encuentros informales (ambas desarrolladas en el CHUVI y no en el CHOU), así como en la elaboración de una revista o boletín interno (que sí se lleva a cabo en el CHOU, pero no en el CHUVI).

C) Como principales funciones de Comunicación al Usuario, no encontramos ninguna diferencia. En ambos casos, se basan en la redacción de materiales de información general y en la supervisión de los puntos informativos y de la señaléctica del hospital.

Respecto de las horas de trabajo en Comunicación Externa, Interna y al Usuario, resulta difícil asignar un reparto horario puesto que las funciones de Comunicación Externa y Comunicación Interna las realizan personas distintas. La responsable de la Comunicación Externa del CHUVI, que además lleva la Comunicación Externa de las otras entidades ya indicadas, reconoce que el $70 \%$ de su tiempo de trabajo lo dedica a la comunicación externa del hospital vigués. La responsable de Comunicación Externa del CHOU coincide también en que más de la mitad de su tiempo de trabajo lo dedica a la comunicación externa del hospital de la capital orensana.

En Comunicación interna, invierte 7 horas diarias el periodista ubicado en el CHOU y 4, la persona instalada en el CHUVI. La Comunicación al Usuario resulta aquella que menos tiempo de trabajo le exige al Gabinete. Una hora por día afirma dedicarle la persona del CHOU y la persona que se encuentra en el CHUVI la incluye dentro de las 4 horas de Comunicación Interna (el tiempo restante estaría al servicio de las demandas de comunicación externa).

Para las personas que se ocupan de la Comunicación Externa ésta es la actividad más importante del Gabinete, la Interna para quien trabaja desde dentro del hospital. La importancia de la comunicación al usuario, a pesar del escaso tiempo que se reconoce se le dedica, recibe un 8 en importancia en ambos casos.

Respecto de la relación entre el gabinete de comunicación y los puntos informativos del hospital, en el caso del CHOU son un total de 4, los cuales dependen del Servicio de Atención al Paciente (SAP) que, a su vez, depende de la Gerencia del hospital. En el caso del CHUVI, los puntos informativos (un total de 5) dependen del Gabinete de Comunicación, mientras que el SAP depende igualmente de Gerencia. En ambos casos, el Gabinete de Comunicación y el Servicio de Atención al Paciente mantienen una relación independiente orgánica y funcionalmente aunque basada en compartir información.

\subsubsection{CHUAC y CHUS: Gabinete interno bipersonal}

El Complejo Hospitalario Universitario de A Coruña (CHUAC) y el Complejo Hospitalario Universitario de Santiago de Compostela (CHUS) son los dos casos en los que el gabinete, de partida, está en una mejor situación para la gestión de la comunicación del Complejo Hospitalario por dos motivos, fundamentalmente: 
Su ubicación: Ambos están integrados en las instalaciones del Complejo Hospitalario. En el caso del CHUAC, el gabinete está situado en la sexta planta del Hotel de Pacientes; en el caso del CHUS, el gabinete se sitúa en la planta baja del Hospital Clínico Universitario.

- $\quad$ La dotación de recursos humanos para una única entidad: Dos personas que trabajan conjuntamente en la misma actividad y para una única entidad.

En relación con su denominación, en el caso del CHUS el nombre oficial es el de "Gabinete de Comunicación e Imagen del Complejo Hospitalario Universitario de Santiago de Compostela" y en el caso del CHUAC es el de "Gabinete de Comunicación del Complejo Hospitalario Universitario de A Coruña”.

Si nos detenemos en las funciones que llevan a cabo, podemos observar las siguientes convergencias:

A) Comunicación Externa. Las funciones de Comunicación Externa son semejantes en uno u otro caso y se basan en las relaciones con los distintos públicos externos del hospital.

B) Comunicación Interna. En el caso de la comunicación interna, las diferencias aparecen en la formación en comunicación del personal, en la planificación de reuniones informativas y en el mantenimiento de los tablones de anuncios (funciones del gabinete del CHUAC que no comparte el del CHUS).

C) Comunicación al Usuario. Respecto de las funciones de Comunicación al usuario, la principal diferencia radica en que el gabinete del CHUS no supervisa los puntos informativos del hospital.

En cuanto a la importancia del trabajo del gabinete en Comunicación Externa y Comunicación Interna, el Gabinete del CHUAC le otorga la nota más alta (un 9) a la Comunicación Externa, un notable (un 8) a la Comunicación Interna y un aprobado (un 6) a la Comunicación al Usuario. El Gabinete del CHUS ubica la importancia de su trabajo en la máxima medida (un 10) en los dos primeros casos y opta por no puntuar la Comunicación al Usuario porque considera que no forma parte de sus competencias directas.

Respecto de los puntos informativos de los Complejos Hospitalarios, en el caso del CHUAC existen dos oficinas del Servicio de Atención al Paciente y un total de nueve puntos informativos. Ambos dependen de Gerencia. En el caso del CHUS, existen también dos oficinas del Servicio de Atención al Paciente, que dependen de Gerencia, mientras que en el caso de los puntos informativos del hospital, hay un mínimo de cinco en el Complejo, que tampoco dependen del gabinete de comunicación.

Por cuanto a la relación entre los Servicios de Atención al Paciente y los Departamentos de Comunicación, según el Gabinete del CHUAC comparten información cuando es necesario y según el Gabinete del CHUS se coordinan puntualmente en determinados momentos. 


\subsubsection{Arquitecto-Marcide y CHOP: Gabinete interno unipersonal}

El Complejo Hospitalario Arquitecto Marcide-Nóvoa Santos cuenta con un departamento de comunicación desde septiembre de 2008. Una periodista es quien se ocupa de la comunicación del Complejo hospitalario, así como de la comunicación de la gerencia de Atención Primaria y del Hospital de la Defensa de Ferrol. Por este motivo, su denominación es la de "Gabinete de comunicación del área sanitaria de Ferrol”. Su emplazamiento está, no obstante, en el Complejo hospitalario ferrolano.

En el caso del Complejo Hospitalario de Pontevedra (CHOP), hay una persona que se ocupa de la comunicación del Complejo, que no pertenece al cuadro de personal del hospital, sino que el servicio está contratado a una consultoría privada. Aún así, la totalidad de su jornada laboral se desarrolla en las instalaciones del Complejo Hospitalario pontevedrés. Por ese motivo, considera que el gabinete se denomina "Comunicación Corporativa".

Para el departamento de comunicación del Complejo Hospitalario Arquitecto Marcide-Nóvoa Santos, la comunicación externa es la prioridad del gabinete y a esta actividad se dedica el $70 \%$ del trabajo del gabinete; el 30\% restante es para Comunicación Interna, donde se incluye la comunicación al usuario. Así se refleja también en la calificación que se le da a la importancia del trabajo del gabinete respecto de: Comunicación Externa (que recibe un 10, la máxima puntuación); Comunicación Interna (un 5) y Comunicación al usuario (un 4). Hay que tener en cuenta la reciente creación de este departamento.

El gabinete del Complejo Hospitalario de Pontevedra se dedica en un 50\% a Comunicación Externa y Comunicación Interna (reciben un 9 y un 10, respectivamente, en la puntuación de la importancia del trabajo del gabinete en estas dos actividades $^{11}$ ), mientras que la comunicación al usuario no es contemplada directamente entre las actividades y competencias del gabinete.

Respecto de sus funciones en estas tres áreas, los gabinetes de comunicación de ambos hospitales realizan las siguientes funciones:

A) Comunicación externa, realizando las funciones habituales de comunicación con los públicos externos, a excepción, en ambos casos, de la actualización de las páginas web de ambos complejos.

B) Comunicación interna. El gabinete de comunicación del Complejo Hospitalario Arquitecto-Marcide todavía no desarrolló muchas de las funciones propias de este ámbito. Hay que destacar que el gabinete del CHOP también elabora un boletín de comunicación interno.

11 Constituye el único caso en el que la Comunicación Interna es puntuada por encima de la Comunicación Externa. 
C) Comunicación al usuario: El gabinete del CHOP sólo reconoce como función propia en este ámbito, la de supervisión de los puntos informativos del hospital.

El Servicio de Atención al Paciente del CHOP cuenta con una oficina en el complejo pontevedrés que depende jerárquicamente de Gerencia. En el Arquitecto Marcide-Nóvoa Santos, existe también una oficina de Atención al Paciente que, en este caso, depende del Servicio de Admisión.

Los puntos informativos del CHOP, un total de 5, dependen del Servicio de Atención al Paciente. El único punto informativo del Complejo Hospitalario Arquitecto-Marcide Nóvoa Santos, depende de Gerencia.

La relación entre el SAP y el Gabinete de Comunicación del CHOP indica que ambos departamentos comparten información. En el caso del Complejo Hospitalario Arquitecto Marcide, desde el gabinete reconocen que no existe ninguna relación entre ambos servicios y que ambos departamentos funcionan de modo independiente.

\subsubsection{Hospitales públicos sin gabinete de comunicación interno}

Los hospitales de Barbanza, Virxe da Xunqueira y el Hospital da Defensa de Ferrol en la provincia de A Coruña; el Hospital da Costa, el Hospital Comarcal de Monforte y el Complejo Hospitalario Xeral-Calde, en la provincia de Lugo; el Hospital de Verín y el Hospital Comarcal de Valdeorras, en la de Ourense y el Hospital del Salnés en la de Pontevedra carecen de gabinete de comunicación interno, es decir, no hay ningún departamento de Comunicación ubicado en sus dependencias ${ }^{12}$. De su comunicación, se ocupan los gabinetes de comunicación de las Direcciones Provinciales o el gabinete central de la Consejería de Sanidad-Sergas, según la zona:

El gabinete de comunicación de la Consejería -Sergas ubicado en Compostela lleva la Comunicación Externa del Hospital da Barbanza y del Hospital Virxe da Xunqueira. Sólo la comunicación externa y nada de comunicación interna ni al usuario.

- $\quad$ Respecto del gabinete de comunicación que se ocupa de los Hospitales de Verín y Valdeorras (que, como quedó indicado, es el mismo que lleva la Comunicación Externa del CHOU, de la Dirección Xeral del Sergas, de la Consejería de Sanidad y de la Gerencia de Atención primaria en Ourense), reconoce que sus funciones comunicativas en estos hospitales orensanos las desempeña en el ámbito de la Comunicación Externa. En el Hospital de Valdeorras, ha realizado algunas actividades puntuales de Comunicación Interna (sobre todo de organización de eventos) y en el de Verín, prácticamente ninguna.

12 La situación descrita corresponde a septiembre de 2009. 
Respecto del gabinete de comunicación de la Dirección provincial del SERGAS en Lugo, que se ocupa de la comunicación fundamentalmente externa de los tres hospitales lucenses, compatibiliza esta actividad con la comunicación externa relativa a la Dirección provincial del SERGAS en Lugo, a la Delegación Provincial de la Consejería de Sanidad y a la gerencia de Atención Primaria en Lugo. La mayor parte de su tiempo de trabajo, lo dedica consecuentemente a Comunicación Externa (siete horas diarias), a Comunicación Interna, las tres horas restantes. La importancia del trabajo del gabinete en las tres vertientes comunicativas también varía: un nueve, un ocho y un seis respectivamente.

Existe un Servicio de Atención al Paciente en cada uno de los tres hospitales de la provincia de Lugo, que dependen de Gerencia (en el caso del Xeral-Calde y del Hospital da Costa) y del Servicio de Admisión, en el caso del Hospital de Monforte.

El Gabinete de Comunicación afirma que no existe ninguna relación con los Servicios de Atención al Paciente de los tres hospitales, sino que funcionan de manera independiente.

Los puntos informativos (uno en cada hospital), dependen en los tres casos del Servicio de Admisión.

En el caso del Hospital de Verín, existe una oficina del Servicio de Atención al Paciente que depende de Gerencia y un punto de información, que depende del Servicio de Admisión y Documentación Clínica.

En el caso del Hospital de Valdeorras, disponen de una oficina del Servicio de Atención al Paciente, que depende de Gerencia y de un punto de información que depende de la Dirección de Gestión y Recursos Humanos.

Tampoco existe ninguna relación funcional ni jerárquica entre el Gabinete de Comunicación y los Servicios de Atención al Paciente de los hospitales orensanos.

\section{Resultados y conclusiones. Líneas de investigación abiertas}

A raíz de la contextualización previamente expuesta, de los datos obtenidos y de su interpretación, podemos apuntar algunas consideraciones acerca del ámbito de la comunicación hospitalaria pública en Galicia:

\subsection{Sector en crecimiento}

Aunque el primer departamento de comunicación hospitalario en Galicia se crea a finales de los años ochenta, es a raíz de la década de los noventa cuando se va detectando su necesidad en otras organizaciones hospitalarias públicas, todas ellas complejos hospitalarios. El hecho de que todos los complejos hospitalarios públicos cuenten en su estructura con un gabinete de comunicación abre el camino a los demás hospitales públicos, que, aunque cuentan con menor cantidad de 
personal y dan servicio a un número más reducido de usuarios, ya comienzan a detectar necesidades comunicativas que están solventando a través de los departamentos de comunicación de la estructura del Sergas-Consejería de Sanidad.

\subsection{Recursos Humanos escasos. La especialización responde a la formación continuada}

En general, los recursos humanos de los hospitales con gabinete (propio o externo) son pocos (alternan la presencia de una o dos personas, con la excepción del Gabinete de la Consejería en el que trabajan cuatro personas, incluido el Jefe de Gabinete), lo que repercute necesariamente en las actividades que llevan a cabo. Cabe señalar que los complejos hospitalarios están compuestos de varios hospitales, muchas veces físicamente alejados, así que en realidad esas dos personas están llevando el peso de la comunicación de todos los centros hospitalarios que forman el complejo.

En cuanto a su formación, predominan los titulados en Periodismo/ Ciencias de la Información. Respecto de su especialización en temas de medicina y salud, la especialización derivó mayoritariamente del trabajo diario y del interés 'a posteriori' por cursos y jornadas del ámbito temático, es decir, lo que se denomina formación continuada.

Tabla II. Especialización de los profesionales de los gabinetes de los hospitales y del SERGAS.

\begin{tabular}{|l|l|}
\hline Tipo de especialización & No Personas \\
\hline Máster/ Posgrado & 0 \\
\hline Curso/ Jornadas & 12 \\
\hline Trabajo previo en editoriales médicas & 1 \\
\hline Ninguna & 2 \\
\hline Total & 15 \\
\hline
\end{tabular}

Fuente: Elaboración propia.

\subsection{Se prioriza la Comunicación Externa}

Principalmente, los gabinetes de los hospitales públicos (propios o externos) se dedican sobre todo a las relaciones con los públicos externos -destacando, los medios de comunicación-y por lo tanto a la parte más visible de su actividad. El grado de desarrollo de la función de comunicación interna en los hospitales públicos se limita a aquellos que tienen gabinete dentro del hospital, que por otra parte son los de mayor tamaño y de mayor número de empleados.

Si tenemos en cuenta las medias de las puntuaciones otorgadas por los gabinetes de los hospitales públicos a la importancia de su trabajo en las tres vertientes de la comunicación institucional, repararemos en la clara apuesta que se realiza por la Comunicación Externa, es decir, la autovaloración que realizan sus profesionales del trabajo que desarrollan muestran la clara importancia que tienen las acciones y estrategias que desarrollan en Comunicación Externa. 
Tabla III. Resumen de las funciones de los departamentos de comunicación hospitalarios en sus tres vertientes: comunicación externa, interna y al usuario.

\begin{tabular}{|c|c|c|c|c|c|c|c|}
\hline Función & CHUVI & $\mathrm{CHOU}$ & CHUS & CHUAC & $\mathrm{CHOP}$ & Marcide & $\begin{array}{l}\text { Xeral- } \\
\text { Calde }\end{array}$ \\
\hline \multicolumn{8}{|l|}{ Comunicación Externa } \\
\hline Relación con los medios de comunicación & $\mathrm{x}$ & $\mathrm{x}$ & $\mathrm{x}$ & $\mathrm{x}$ & $\mathrm{x}$ & $\mathrm{x}$ & $\mathrm{x}$ \\
\hline Relación con otras instituciones & $\mathrm{x}$ & $\mathrm{x}$ & $\mathrm{x}$ & $\mathrm{x}$ & $\mathrm{x}$ & $\mathrm{x}$ & $\mathrm{x}$ \\
\hline Relación con asociaciones de pacientes & & $\mathrm{x}$ & $\mathrm{x}$ & $\mathrm{x}$ & $\mathrm{x}$ & $\mathrm{x}$ & \\
\hline $\begin{array}{l}\text { Actualización de las informaciones de la página web } \\
\text { del hospital }\end{array}$ & $\mathrm{x}$ & $\mathrm{x}$ & $\mathrm{x}$ & $\mathrm{x}$ & & & $\mathrm{x}$ \\
\hline Manual de imagen corporativa & $\mathrm{x}$ & $\mathrm{x}$ & & $\mathrm{x}$ & $\mathrm{x}$ & $\mathrm{x}$ & $\mathrm{x}$ \\
\hline Organización de actos/ eventos & $\mathrm{x}$ & $\mathrm{x}$ & $\mathrm{x}$ & $\mathrm{x}$ & $\mathrm{x}$ & $\mathrm{x}$ & $\mathrm{x}$ \\
\hline Inserción de publicidad & & & & & & $\mathrm{x}$ & $\mathrm{x}$ \\
\hline \multicolumn{8}{|l|}{ Comunicación Interna } \\
\hline Planificación de reuniones informativas & $\mathrm{x}$ & $\mathrm{x}$ & & $\mathrm{x}$ & $\mathrm{x}$ & $\mathrm{x}$ & $\mathrm{x}$ \\
\hline Planificación de encuentros informales & $\mathrm{x}$ & & $\mathrm{x}$ & $\mathrm{x}$ & $\mathrm{x}$ & & \\
\hline Actualización de contenidos en la Intranet & $\mathrm{x}$ & $\mathrm{x}$ & $\mathrm{x}$ & $\mathrm{x}$ & $\mathrm{x}$ & $\mathrm{x}$ & $\mathrm{x}$ \\
\hline Formación en comunicación del personal & $\mathrm{x}$ & & & $\mathrm{x}$ & $\mathrm{x}$ & $\mathrm{x}$ & \\
\hline Manual de acogida a nuevos profesionales & $\mathrm{x}$ & $\mathrm{x}$ & $\mathrm{x}$ & $\mathrm{x}$ & $\mathrm{x}$ & & \\
\hline Tablón de anuncios & $\mathrm{x}$ & $\mathrm{x}$ & $\mathrm{x}$ & $\mathrm{x}$ & $\mathrm{x}$ & & $\mathrm{x}$ \\
\hline Buzón de sugerencias & $\mathrm{x}$ & $\mathrm{x}$ & & & $\mathrm{x}$ & & \\
\hline Documentos internos & $\mathrm{x}$ & $\mathrm{x}$ & $\mathrm{x}$ & $\mathrm{x}$ & $\mathrm{x}$ & & $\mathrm{x}$ \\
\hline Revista/Boletín interno & & $\mathrm{x}$ & $\mathrm{x}$ & $\mathrm{x}$ & $\mathrm{x}$ & & \\
\hline \multicolumn{8}{|l|}{ Comunicación con el Usuario } \\
\hline $\begin{array}{l}\text { Redacción de materiales de información general: guía } \\
\text { de acogida, campañas preventivas, etc. }\end{array}$ & $\mathrm{x}$ & $\mathrm{x}$ & $\mathrm{x}$ & $\mathrm{x}$ & $\mathrm{x}$ (ayuda) & $\mathrm{x}$ & $\mathrm{x}$ \\
\hline
\end{tabular}




\begin{tabular}{|l|l|l|l|l|l|l|l|}
\hline $\begin{array}{l}\text { Redacción de materiales de información específica: } \\
\text { formularios de Consentimiento Informado, instruc- } \\
\text { ciones médicas, etc. }\end{array}$ & & & & & & & \\
\hline Supervisión de puntos informativos del Hospital & $\mathrm{x}$ & $\mathrm{x}$ & & $\mathrm{x}$ & $\mathrm{x}$ & $\mathrm{x}$ & \\
\hline Supervisión del Servicio de Atención al Paciente & & & & & & & \\
\hline Supervisión de la señaléctica del centro & $\mathrm{x}$ & $\mathrm{x}$ & $\mathrm{x}$ & $\mathrm{x}$ & & $\mathrm{x}$ & $\mathrm{x}$ \\
\hline Revista/Boletín para pacientes & & & & & & & \\
\hline
\end{tabular}

Fuente: Elaboración propia.

\subsection{La de Comunicación al Usuario es la vertiente menos atendida}

El tiempo que se le dedica a la comunicación al usuario es inferior al invertido en comunicación externa o interna. Cuantitativamente, el tiempo dedicado a comunicación externa es superior, seguido de la comunicación interna.

Figura 3. Tiempo de trabajo del gabinete dedicado a comunicación externa, interna y al usuario

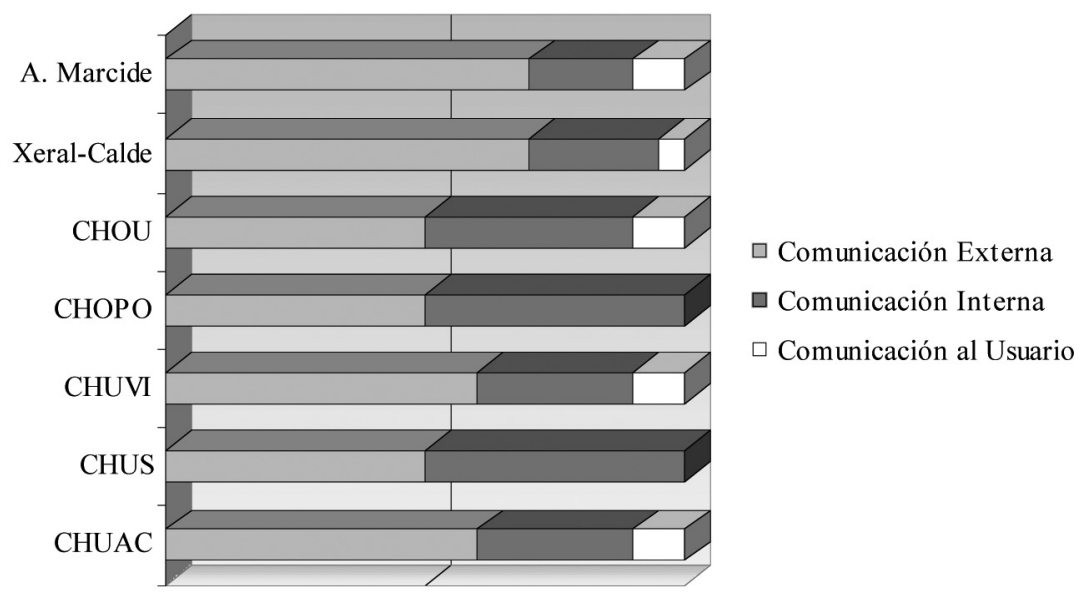

Fuente: Elaboración propia. 


\subsection{Todavía falta una apuesta estratégica por la función de Comunicación}

Ni los Servicios de Atención al Paciente ni los Puntos Informativos de los hospitales dependen de los Departamentos de Comunicación.

Los Servicios de Atención al Paciente dependen, mayoritariamente, bien de la Gerencia, bien del Servicio de Admisión, sin otro tipo de vinculación con el Gabinete más allá de la de compartir información, algo que no sucede ni en todos los casos ni de forma continuada. En ningún hospital, el departamento de comunicación se ocupa de supervisar la función de los servicios de atención al paciente, aunque tienen en la comunicación una herramienta indispensable en la gestión de las sugerencias de los pacientes.

Tampoco los puntos informativos de los hospitales dependen mayoritariamente de los Gabinetes de Comunicación (esto sólo sucede en el caso del Complejo Hospitalario Universitario de Vigo), a pesar de que la mayoría de los gabinetes ubican entre sus funciones la supervisión de los puntos informativos del hospital. Si el gabinete los supervisa, ¿̇por qué no dependen de él en el organigrama del hospital?

\section{6. ¿Un modelo utópico?}

Tras el acercamiento a la realidad del sector, el modelo propuesto de Departamento de Comunicación para un hospital revela que es en la Comunicación con el Usuario, donde puede plantearse una mayor indefinición acerca de las funciones o canales de comunicación que debe establecer el gabinete.

Los documentos de información específica (como los consentimientos informados) se dejan en las manos de los expertos médicos, a pesar de que son documentos informativos que se dirigen a los usuarios. Los puntos de información no siempre son supervisados por el gabinete, menos aún el Servicio de Atención al Paciente. Tampoco se edita revista/ boletín para pacientes.

Es probable que sea necesaria una reflexión acerca del papel que debe cumplir el departamento de comunicación hospitalario con un público intermedio (no es externo, pero tampoco puede considerarse interno) tan importante como los usuarios.

En conclusión, podemos apuntar que la comunicación hospitalaria pública en Galicia es un sector en expansión, por lo que es probable que en un futuro más o menos próximo los hospitales que carecen del mismo decidan apostar por la creación de un departamento interno que vehiculice la función de comunicación.

Dado que los recursos humanos con los que cuentan los gabinetes son escasos, la actividad prioritaria es la de comunicación externa, quedando relegada en importancia su función directiva en el caso de la comunicación con los usuarios 
efectivos de los servicios del hospital. Una manera de apostar por dicho rol estratégico, sería la de establecer jerárquicamente la dependencia de los departamentos de atención al paciente (que puede compartir la dependencia con Gerencia para dar solución real a los problemas de gestión que se vayan detectando) y de los puntos informativos del hospital (que sin duda realizan una labor informativa muy importante).

Para conocer cómo se encuentra el sector a nivel de España, resultaría conveniente que se llevasen a cabo investigaciones o estudios a nivel estatal o de otras comunidades autónomas, de los que, en estos momentos, carecemos para poder realizar comparativas.

\section{Referencias bibliográficas}

Asenjo Sebastián, M. A. (di) (2006): Gestión diaria del hospital. Barcelona: Masson.

Asociación de la Comunicación Sanitaria y Social (ACSAS) (1999): “Comunicación hospitalaria”, Todo hospital, nº 154, pp. 99-100.

Corella, J. M. (1998): Introducción a la gestión del marketing en los servicios de salud. Pamplona: Departamento de Salud, Gobierno de Navarra.

Costa, J. (1995): Comunicación Corporativa y Revolución de los servicios. Madrid: Ciencias Sociales.

Font, D. (2006): “Organización general del hospital moderno” en Asenjo Sebastián, M. A. (dir.): Gestión diaria del hospital. Barcelona: Masson, pp. 383-396.

González Borjas, A. (2004): “Salud, información periodística especializada en alza”, Ámbitos, n. 11-12, pp. 301-310.

Kuteev-Moreira, P. (2003): “O Gabinete de Comunicação no Hospital”, Comunicação Empresarial, nº 25, pp. 4-9.

March Cerdá, J. C. et al. (2002): "Marketing y comunicación interna en hospitales y en atención primaria: opinión de profesionales y de gestores”, Horizonte sanitario, no 1, pp. 6-17.

Martínez Ortuño, I. (1994): “Modelo comunicativo para un hospital”, en Orive Riva, P.: Comunicación sanitaria, Madrid: Dossat 2000, pp. 78-83.

Pereira, R. (2006): "La comunicación institucional de la salud en el ámbito local. El caso del complejo hospitalario universitario de Santiago de Compostela”, en López Rita, R.; Fernández Beltrán, F. y Durán Mañes, A.: La comunicación corporativa en el ámbito local. Castellón: Publicacions de la Universitat Jaume I.

Ruiz, A (1990): Marketing de servicios. Buenos Aires: Tesis.

Valls Roig, A. (1999): “Inteligencia emocional para el cambio organizativo”, en Silió Villamil, F. y Solas Gaspar, O. (ed.): Nuevas perspectivas en la gestión estratégica de los recursos humanos en organizaciones sanitarias. Granada: EASP, pp. $128-143$. 